\title{
NORMAS Y RAZONAMIENTO PRÁCTICO. UNA CRÍTICA A JOSEPH RAZ
}

(I)

\begin{abstract}
- os teóricos del derecho han reflexionado persistentemente sobre la normatividad y naturaleza de los sistemas jurídicos ${ }^{1}$; temas que pueden ser considerados de principal importancia para una teoría general del derecho. Una manera de abordar estos problemas es establecer el significado de los enunciados jurídicos y esclarecer las razones de los sujetos para emplear un lenguaje normativo ${ }^{2}$. Respecto a esto, las teorías positivistas pueden clasificarse en:
\end{abstract}

(i) Teorías reduccionistas ${ }^{3}$ : sin perjuicio de las importantes variantes en esta postura, sostienen la posibilidad de traducir los enunciados jurídicos a meros enunciados de hecho, vgr.: enunciados de probabilidad de sufrir un daño.

${ }^{1}$ Raz, Joseph: «The Problem About The Nature Of Law» en Contemporary Philosophy. A New Survey, vol. 3, pp, 107-125, 1982, M. Nijhoff Publishers, Boston, Londres. 1981.

${ }^{2}$ Raz, Joseph: «La Pureza de la Teoría Pura» en Análisis Filosófico, vol. 1, n. ${ }^{\circledR} 1$, pp. 71-88,

Raz, Joseph: «La Teoría de Kelsen de la Norma Fundamental» en La Autoridad del Derecho, pp. 157-187, 1982, UNAM, trad. por R. Tamayo, México.

Para una versión alternativa, cfr.:

Bulygin, Eugenio: «Norms, Normative Propositions, and Legal Statements» en Contemporary Philosophy. A New Survey, vol. 3, pp. 127-152, 1982, M. Nijhoffs Publishers, Boston, Londres.

Bulygin, Eugenio: «Enunciados Jurídicos y Positivismo Jurídico. Respuesta a Raz» en Análisis Filosófico, vol. 1, n. ${ }^{\circ}$ 2, pp. 49-59, 1981.

${ }^{3}$ Hart, Herbert: «Scandinavian Realism» en Essays in Jurisprudence and Philosophy, pp. 161-170, 1988, Clarendon Press, Oxford. Press, Oxford.

Hart, Herbert: «Legal Duty and Obligation» en Essays on Bentham, pp. 144, 1982, Clarendon

MacCormick, Neil: «On Analytical Jurisprudence» en An Institucional Theory of Law, pp. 103 y ss., 1986, Reidel PC, Dordrecht, Boston.

Ross, Alf «Sobre el Derecho y la Justicia», cap. 2, 1963, EUDEBA, trad. por G. Carrió, Buenos Aires. 
(ii) Teorías no-reduccionistas ${ }^{4}:$ niegan que el significado de los enunciados jurídicos se agote al especificar sus condiciones de verdad. Al igual que en las teorías reduccionistas, las discrepancias entre las diferentes teorías no-reduccionistas son frecuentes ${ }^{5}$.

En este trabajo consideraremos solamente una discusión pertinente a las teorías no-reduccionistas. Para ello analizaremos la teoría de Joseph Raz: las normas jurídicas como razones para actuar. Según esta teoría, la normatividad del derecho tiene que explicarse de tal manera que posibilite: a) afirmar que los enunciados jurídicos implican enunciados de razones para actuar; y b) señalar el tipo específico de razón que constituyen las normas jurídicas ${ }^{6}$.

Considerando de manera principal al libro «Practical Reason and Norms» ${ }^{7}$, reflexionaremos acerca de algunos temas centrales de la teoría de Raz, e intentaremos mostrar la necesidad de revisar los siguientes puntos:

- los criterios de distinción entre razones de primer y segundo orden;

- las ventajas de la teoría de Raz respecto de la teoría práctica de las normas atribuida a $\mathrm{Hart}^{8}$;

- la naturaleza de las razones excluyentes. Barcelona.

${ }^{4}$ Kliemt, Hartmut: «Instituciones Morales», pp. 186 y ss., 1986, Alfa, trad. por J. M. Seña,

Raz, Joseph: «La Validez Jurídica» en La Autoridad del Derecho (cit. nota 2), pp. 187-203. Backer, G.: «Defeasibility and Meaning» en Law, Morality and Society. Essays in Honour of H. L. A. Hart, pp. 26-57, Raz y Haecker (eds.), 1977,

Clarendon Press, Oxford. pp. 286-308.

${ }^{5}$ Hart, Herbert: «Kelsen Visited» en Essays in Jusrisprudence and Philosophy (cit. nota 3),

${ }^{6}$ Raz, Joseph: «Reasons for Action, Decisions and Norms» en Mind (1975), pp. 481-500.

Nino, Carlos: «Normas Jurídicas y Razones para la Acción» en La Validez del Derecho, pp. 126-143, 1985, Astrea, Buenos Aires.

${ }^{7}$ Raz, Joseph: «Practical Reason and Norms», segunda edición, 1990, Hutchinson of London, Londres (en adelante, PRN).

${ }^{8}$ Hart ha propuesto un análisis acerca de las normas como razones autoritativas y ha criticado la teoría de Raz en «Commands an authoritative Reasons» en Essays on Bentham, ob. cit., pp. 243268- Cf- «Sovereignity and Legally Limited Goverment» en Essays on Bentham, ob cit., p. 239. 


\section{(II)}

La clasificación de las razones propuesta por Raz constituye la base de sus dos modelos de racionalidad. El Modelo 1 se configura cuando el agente obra de acuerdo con el balance de razones de primer orden (en adelante «RPO»), cuyo resultado constituye una razón concluyente. El Modelo 2 se configura cuando el agente actúa con fundamento en una razón excluyente (en adelante «RET»). La posibilidad de identificar ambos modelos depende de la distinción entre las RPO y las RET. Esa distinción es relativa al papel que desempeñan en el razonamiento práctico. Por lo tanto, es necesario analizar la estructura de este tipo de razonamiento.

Según Raz, todo hecho descripto en una inferencia práctica, cuyas premisas no sean redundantes, es considerado una razón para la acción descripta en la conclusión. Debe distinguirse dentro de esta inferencia, la descripción de aquellos hechos respecto de los cuales creer en su existencia implica que el agente tiene una actitud crítico práctica. Son ellos los únicos que pueden figurar como premisa mayor del argumento, constituyendo la razón operativa para la acción. Las razones auxiliares constituyen la clase complementaria de las razones operativas y su función es transferir la actitud práctica desde la premisa mayor a la conclusión ${ }^{9}$. La inferencia práctica, descripta por Raz, tiene por conclusión un enunciado deóntico; y si bien él admite que hay por lo menos dos tipos básicos de inferencias prácticas, sólo da elementos para reconstruir una de ellas. Esto es, aquel argumento que responde a qué acción tenemos una razón (prima facie) para realizar ${ }^{10}$; donde la premisa mayor describe o bien un valor, un interés, un deseo, o una norma; la premisa menor describe la acción que el agente considera suficiente para satisfacer lo expresado por la premisa mayor, y la conclusión enuncia que se debe realizar la acción señalada. En palabras equivalentes para Raz, la conclusión enuncia que hay razones para realizar tal acción.

El diferente papel que las razones juegan en el razonamiento se apoya, en el caso de las RPO, en que ellas se relacionan en virtud de su fuerza o peso. El mayor o menor peso de estas razones está determinado por los valores a los que ellas sirven ${ }^{11} \mathrm{y}$

\footnotetext{
${ }^{9}$ Raz, Joseph, PRN, p. 33.

${ }^{10}$ Raz, Joseph: «Introducción», en Razonamiento Práctico, pp. 14-21, Fondo de Cultura Económica, trad. por J. Utrilla, 1986, México.

${ }^{11}$ Raz, Joseph, PRN, p. 77.
} 
el balance entre ellas define el modelo 1 de racionalidad. Respecto de las RET, Raz no menciona ninguna propiedad que las defina y en base a la cual se explique su diferente funcionamiento. Ellas se caracterizan por operar de hecho en las consideraciones del agente, excluyendo el modelo 1 de racionalidad y justificando la acción en forma independiente de éste. Es necesario precisar el concepto de balance de razones ya que las RET no excluyen todo balance de razones, sino aquel que define el modelo 1 de racionalidad. La aceptación de una razón excluyente no elimina:

a) al balance de razones de segundo orden

b) al balance entre RET y RPO no vencidas

En estos últimos dos casos, no es verdad sostener que una RET excluye el balance de razones ${ }^{12}$. En adelante siempre nos referiremos al balance de razones en relación al modelo 1 de racionalidad que resulta excluido por una RET.

Nuestro interés se centra principalmente en la teoría de las normas que sostiene $\mathrm{Raz}^{13}$, y dado que ellas constituyen RET, reflexionaremos acerca de algunas afirmaciones de este autor que nos permitan esclarecer el concepto de RET. De manera especial hay que tener en cuenta que, según Raz, las RET implican una manera independiente de justificar la acción ${ }^{14}$, característica de los casos en que los sujetos siguen normas; de allí infiere Raz que las normas son $\mathrm{RET}^{15}$.

\section{(III)}

Al explicar las normas como RET, Raz advierte que no cualquier exclusión del balance de razones da lugar a una acción racional del agente. Dice: «Pero no debemos confundir una decisión arbitraria de no considerar razones y no actuar en base a ellas con una razón para excluir razones y no actuar en base a ellas» ${ }^{16}$. Inmediatamente, explica que sólo si la razón (que excluye) es válida o si la regla es justificada, la acción del agente es racional y puede decirse que obró en base a una RET. Debe advertirse que, a diferencia de las RPO, las RET son - por

${ }^{12}$ Raz, Joseph, PRN, pp. 46, 47.

${ }^{13}$ Nino, Carlos: «El Concepto de Validez Jurídica en la Teoría de Kelsen» en La Validez del Derecho (cit. nota 6), p. 26.

${ }^{14}$ Raz, Joseph, PRN, pp. 40-45.

${ }^{15}$ Raz, Joseph, PRN, p. 194.

${ }^{16}$ Raz, Joseph, PRN, pp. 61, 62. 
definición- válidas. Por lo tanto, aunque de hecho se excluya el balance de razones, la acción de un agente sólo será considerada racional cuando la exclusión (de razones válidas) ${ }^{17}$ se base en una razón válida ${ }^{18}$. De este modo, un proceder con fundamento en una RET se distingue de un proceder arbitrario si el agente está justificado en excluir el balance de razones $^{19}$.

El problema que nos interesa destacar es que Raz no explicita un criterio general que permita distinguir las exclusiones justificadas de las injustificadas; en otras palabras, no proporciona un criterio general para distinguir acciones arbitrarias de aquellas basadas en una RET. La metodología de Raz para estos casos consiste en presentar ejemplos en los cuales, según su opinión, está justificada la exclusión del balance de razones ${ }^{20}$ (ahorro de tiempo, de trabajo y coordinación de actividades). Juzgar si está justificada la exclusión supone la existencia de patrones de evaluación que permitan clasificar ciertas acciones y/o estados de cosas como justificados o correctos, a pesar de lo que indica el balance de razones. Esta discriminación entre razones que justifican y razones que no justifican la exclusión implica cotejar razones. Si este cotejo es o no un «balance de razones» en el sentido en que Raz emplea este término depende, nuevamente, de la aplicación de un criterio general de identificación de los dos tipos de razones. En todo caso, es claro que detrás de la exclusión justificada de un balance - esto es, detrás del modelo 2 de racionalidad- existe otro balance que clasifica ciertas razones como RET, es decir como razones buenas o válidas. Esto indica que el modelo 2 de racionalidad, que se supone independiente del modelo 1, es una forma reducida de argumentar. Una vez desarrollada muestra claramente el balance o cotejo de razones que la sustenta, y en

${ }^{17}$ «Es crucial recordar que las razones excluyentes excluyen razones válidas. No es necesario una razón especial para excluir malas razones», Raz, Joseph, PRN, p. 212, note 12.

${ }^{18}$ «... Recuérdese que una razón excluyente tiene éxito en excluir solamente si es válida». Raz, Joseph, PRN, p. 215, note 24.

${ }^{19}$ MacCormick, Neil: «Limits of Rationality in Legal Reasoning» en An Institutional Theory of Law, (cit. nota 3), p. 191.

MacCormick, Neil: «Hart», p. 61 y ss., 1981, Arnold Publishers, Londres. 256.

Hart, Herbert: «Command and Authoritative Legal Reason» en Essays on Bentham (cit. nota 3), p.

${ }^{20}$ Raz trata de justificar estas instituciones en «Rethinking exclusionary reasons. Postscriptum to the second edition», PRN, pp. 186 and 196; y en «Facing up», Southern California Law Review, vol. 62, n. ${ }^{\circ} 3-4$, pp. 1164, 1165, 1989.

Véase también, Moore, Michael «Authority, Law and Razian Reasons», Southern California Law Review, vol. 62, n. ${ }^{\circ}$ 3-4. p. 850, 1989. 
virtud del cual la acción es considerada racional. Adviértase que la independencia entre los dos modelos de racionalidad es uno de los test que propone Raz para identificar una acción basada en una $\mathrm{RET}^{21}$.

Raz sostiene que la naturaleza de las normas consiste en ser, a la vez, una RET y una RPO para la acción que exigen ${ }^{22}$. La no advertencia de este rasgo, junto a la imposibilidad de explicar las normas independientemente de su práctica, es una de las críticas que Raz formula a la teoría práctica de las normas de $\mathrm{H}$. Hart.

Raz sostiene que las RET son aquellas razones que funcionan excluyendo el balance de razones $^{23}$, y si las normas funcionan de este modo sólo cuando el agente las acepta y las sigue, entonces Raz debe admitir que una norma es una RET sólo cuando es aceptada y seguida por el agente. Por lo tanto, es la aceptación y no la existencia de la norma, lo que constituye la RET para la acción ${ }^{24}$. Sostener que la RET es el hecho de que la norma exista, con independencia de su aceptación, exige señalar una propiedad que permita identificar ese tipo de razones ${ }^{25}$. De no ser así, Raz debe admitir, o bien que la aceptación es lo que constituye la RET, o que la existencia de las normas consiste en que ellas son aceptadas y seguidas. En ambos casos queda expuesto a la misma crítica que le formulara a Hart: no poder explicar las normas con independencia de su práctica. Podría argumentarse que aceptar una regla no implica seguirla. En este sentido, Raz habla de reglas

${ }^{21}$ Raz, Joseph, PRN, pp. 43, 44.

${ }^{22}$ Raz, Joseph, PRN, pp. 58 y 59.

Raz, Joseph: «Promises and Obligations» en Law, Morality and Society. Essays in Honour of H. L. A. Hart (cit. nota 4), p. 221 y ss.

${ }^{23}$ Raz, Joseph, PRN, p. 190.

${ }^{24}$ Von Wright, Georg: «Explanation and Understanding of Action en Practical Reason, p. 54, 1983, Cornell UP., Ithaca, New York.

Von Wright, Georg: «Determinism and the Study of Man» en Practical Reason, ob. cit., pp. 35-72. Von Wright, Georg: «An essay on door-knocking», Rechtstheorie, vol. 19, p. 281, 1988.

Alchourrón, Carlos: «Razones y Prescripciones. Discusión» en Análisis Filosófico, vol. I, n. ${ }^{\circ} 1,1981$, pp. 49 y 50. 3), p. 258.

Hart, Herbert: «Command and Authoritative Legal Reason» in Essays on Bentham (cit. nota

Raz, Joseph: «Hart on moral rights and legal duties», Oxford Journal of Legal Studies, vol. 4, p. 123, 1984.

${ }^{25}$ Moore, Michael, «Authority, Law and Razian Reasons», (cit. nota 20), p. 841. 
individuales a las que el agente no sigue por falta de voluntad, tiempo, etc. Sin embargo, dado que el funcionamiento en la práctica es el único criterio ofrecido por Raz para identificar una RET, en el caso de reglas aceptadas pero que no se siguen, ellas no son razones excluyentes. Esto prueba que no todas las reglas lo son y que, sin otros argumentos, la insistencia en el carácter excluyente de las normas es una petición de principio. Si asumimos (como Raz lo hace) que todas las reglas son, por definición, RET entonces no pueden existir reglas que no se sigan, es decir, que no funciones como RET. Por consiguiente, Raz queda nuevamente expuesto a su propia crítica a la teoría práctica de las normas. Con vistas a mantener la distinción entre la existencia de la norma y su aceptación, asumiremos que es esta última la que constituye la RET para la acción. Aceptar una norma es creer que hay razones para seguirla, es tener una razón operativa para hacerlo. Según Raz, seguir una regla implica su aceptación como una razón excluyente para no actuar sobre razones conflictivas aun cuando estas últimas puedan inclinar el balance de razones ${ }^{26}$. De acuerdo a ello podemos concluir que seguir una norma consiste en hacer la acción específica que la norma indica sin considerar las razones de primer orden a favor o en contra de esta última acción. Quien actúa de esta forma, obra en base de una RET: la aceptación de la norma.

Sostendremos aquí que, en virtud de la noción de razonamiento práctico delineado por Raz, si existen diferencias entre las RPO y las RET, éstas no se encuentran en el desarrollo de ese razonamiento. Las razones operativas, dentro de la inferencia práctica, cumplen idéntico papel, sean prima facie, concluyentes o excluyentes. En consecuencia, el papel que desempeñan no puede ser el fundamento de la clasificación.

Consideremos un ejemplo expuesto por $\operatorname{Raz}^{27}$ :

El comandante ha ordenado a Jeremías sustraer, para uso militar, la camioneta de un civil. Jeremías acepta las órdenes del comandante y, por lo tanto, esta aceptación es su razón para seguir sus mandatos y en este caso en particular ello significa que debe apropiarse de la camioneta dejando de lado cualquier otra razón en pro o en contra de esta acción. Supongamos, además,

${ }^{26}$ Raz, Joseph, PRN, p. 61.

${ }^{27}$ Raz, Joseph, PRN, p. 41. 
que Jeremías consiente en que tomar la camioneta es una acción que, conforme al balance de razones, también debería ejecutarse. Él considera que no debe existir diferencia entre propiedad propia y ajena; y que en cualquier circunstancia es bueno poder disponer de todos los bienes que tenemos a nuestro alcance. Él ha sopesado diversos intereses, deseos y valores y ha concluido que tiene una razón concluyente a favor de la acción. En ambos casos, a partir del momento en que Jeremías considera que tiene una razón válida para una determinada acción (sea esta razón la aceptación de un mandato o cualquier otra razón), su razonamiento práctico se desenvuelve de la misma manera: Jeremías debe (tiene razones para) realizar todo aquello que sea suficiente para consumar la acción respectiva.

Siguiendo a Raz, en el primer caso, si Jeremías tiene una razón para seguir las órdenes de su comandante, eso implica que tiene una razón excluyente para sustraer la camioneta, ya que esta última acción es necesaria para seguir la orden del comandante. En el segundo caso, si considera que lo mejor es no hacer distinciones entre bienes propios y ajenos, ésta es una razón concluyente para disponer de cualquier bien; ello implica que, en esta oportunidad, hay una razón para tomar la camioneta.

Cuando Raz propone como test para saber si un agente actúa en base a una RET o una RPO que atendamos a la forma en que éste desarrolla su razonamiento, sin duda se refiere a un modelo de razonamiento distinto al previamente explicado (véase, punto II). La inferencia a la que ahora se refiere consiste en una evaluación previa de las razones en conflicto y cuya resolución (que según Raz depende de un mecanismo lógico) nos dará la premisa mayor de la inferencia práctica anteriormente explicada.

Raz admite expresamente que hay dos puntos de vista desde los cuales pueden ser evaluadas las razones para resolver el conflicto ${ }^{28}$ :

Estos puntos de vista provienen de considerar: PV1) El peso o fuerza de las razones (Modelo 1 de Racionalidad. PV2) Un principio específico de razonamiento práctico que establece que las RET siempre prevalecen sobre las RPO (Modelo 2 de racionalidad).

Es preciso advertir que Raz también denomina razonamiento práctico al proceso de resolución de un conflicto de razones y el papel que en él desempeñan las razones es el criterio de distinción entre una RET y una RPO. Raz no analiza la estructura de este tipo de razonamiento. Con respecto al punto de vista 2 de

${ }^{28}$ Raz, Joseph, PRN, p. 40. 
evaluación (PV2), su aplicación presupone la posibilidad de identificar previamente las RET en base a alguna propiedad distintiva, que Raz en ningún momento señala. Debido a ello, estamos en condiciones de afirmar las siguientes alternativas:

(a) Un razonamiento cuya conclusión se fundamenta en una RET, es decir la resolución de un conflicto de razones con fundamento en una RET no es independiente ni distinto del balance de razones (modelo 1 de racionalidad). Esto puede afirmarse en base a lo anteriormente expuesto: el punto de vista 2 (PV2) de evaluación se presenta sólo de manera abreviada. El desarrollo del argumento muestra que esta perspectiva también reposa en un balance de razones, y este balance no es diferente del balance de razones conforme al peso o fuerza, que constituye el punto de vista 1 (PV1) de evaluación.

(b) La resolución de un conflicto de razones con fundamento en una RET es distinto e independiente del balance de razones sólo si se admite la existencia de patrones evaluativos distintos e independientes de la fuerza o peso en virtud de los cuales es posible clasificar las razones. Según esta interpretación, las razones que se consideran excluyentes no son, en sí mismas, diferentes de las RPO: la misma razón podría constituir alternativamente una RET o una RPO, según sea el punto de vista de evaluación aplicado.

c) La resolución de un conflicto de razones con fundamento en una RET es distinto del balance de razones porque de hecho éste es excluido. En otras palabras, cualquier razón podría ser vista como una RET siempre que en la evaluación del agente funcione de manera excluyente.

Conforme a estas tres interpretaciones posibles, si una norma es una RET, es factible afirmar alguna de las siguientes alternativas:

(a') Una norma es una razón de la misma naturaleza que las RPO, pero su peso es tal que vence excluyendo razones ${ }^{29}$.

(b') Una norma es una razón distinta de una RPO sólo porque a ella se aplica un criterio evaluativo diferente al que da lugar al modelo 1 . Su carácter excluyente radica en la atribución de mayor jerarquía a este criterio evaluativo respecto del que identifica las RPO.

(c') Una norma es una razón distinta de una RPO porque de

${ }^{29}$ Perry, Stephen, «Secon-Order Reasons, Uncertainty and Legal Theory», Southern California Law Review, vol. 62, n. ${ }^{\circ}$ 3-4, p. 932, 1989. 
hecho siempre excluye razones. Si las normas son RET, entonces siempre que existe una norma el agente debe seguirla; ya que el punto de vista 2 (PV2) de evaluación establece que ellas siempre prevalecen.

\section{(VI)}

Lo expuesto hasta aquí demuestra que la clasificación y el significado de los distintos tipos de razones es ajeno al concepto y a la estructura del razonamiento práctico explicado en el punto II y expuesto por Raz en «Practical Reason and Norms». La ambigüedad de esta expresión, que en un sentido amplio puede referirse a cualquier procedimiento por el cual se resuelve un problema práctico, hace plausible las afirmaciones de Raz sobre los supuestos diferentes roles que las razones desempeñan en él.

Los conceptos de razón concluyente y de RET no constituyen una respuesta a la pregunta por qué se debe hacer, sino más bien parecen ser conclusiones acerca de conflictos sobre cuál es la mejor razón. Sin duda Raz incluiría a este punto dentro de los principales temas de una teoría ética y no de una teoría normativa. Que el conflicto planteado sea ético no significa que la estructura de la inferencia práctica y los principios lógicos que la rigen sean problemas que deba resolver la ética. Bien señala Raz que una teoría del razonamiento práctico se ocupa de éste independientemente del contenido ético, político o jurídico de sus premisas. Lo que señalamos aquí es que Raz explica un tipo de razonamiento práctico diferente al involucrado en la resolución del conflicto de razones.

Si hay un tipo de razonamiento que resuelva ese conflicto de razones y cuya conclusión establece cuál es la razón vencedora, ya sea en base a su peso moral o estratégico o en base a cualquier otro patrón, Raz no se detiene en él. Debemos admitir, entonces, que si no está claro el concepto de razonamiento práctico, tampoco es claro hablar del papel que las razones desempeñan en él. La falta de precisión quita solidez al fundamento de la distinción entre los diferentes tipos de razones. Con ello, la explicación de las normas dada por Raz tiene que ser revisada, ya que se asienta sobre la posibilidad de distinguir los tipos de razones antes señalados.

Por último, queda en pie la cuestión de la plausibilidad de denominar «racional» a la acción de un agente porque resuelve el conflicto de razones desde una determinada dimensión evaluativa ${ }^{30}$. Más aun cuando no se proporciona, explícitamente, un fundamento suficiente para otorgar primacía a una dimensión sobre otra.

\footnotetext{
${ }^{30}$ Moore, Michael, «Authority, Law and Consent» (cit. nota 20), p. 883 y ss.
} 